\title{
Introduction of an in-house real-time PCR assay to detect severe acute respiratory syndrome coronavirus-2 (SARS CoV-2) in Sri Lanka: challenges and issues
}

\author{
Vigeetha Withanage ${ }^{1}$, Daniel Chu ${ }^{2}$, Malik Peiris ${ }^{2}$, Jude Jayamaha ${ }^{1 *}$ \\ ${ }^{1}$ Medical Research Institute, Ministry of Health, Sri Lanka; ${ }^{2}$ School of Public Health, University of Hong Kong \\ Correspondence: jayamahacar@gmail.com \\ iD https://orcid.org/0000-0001-5977-8851
}

DOI: https://doi.org/10.4038/jccpsl.v26i5.8345

Received on 17 May 2020

Accepted on 6 June 2020

\section{Summary}

In late December 2019, health officials of China reported a cluster of pneumonia with an unknown aetiology, which was later discovered as a novel coronavirus named severe acute respiratory syndrome coronavirus-2 (SARS-CoV-2) by the World Health Organization (1). Virus spread over the mainland in China with an alarming morbidity and mortality rate. In about two weeks from discovery of the first case, another was reported from Thailand denoting international threat (2-3).

Sri Lanka being one of the main tourist attractions as well as having a significant Chinese work force, country was at a greater risk of acquiring the infection (4-6). On the other hand, the spectrum of disease in human is yet to be determined and signs and symptoms are highly non-specific making it difficult to be recognized solely on clinical grounds (2). In this backdrop, laboratory identification of the infected patients was crucial (7). SARS-CoV-2 is a newly detected virus and established Reverse Transcription Polymerase Chain Reaction (RT-PCR) methods are scarce and establishing an accurate diagnosis is challenging in low resource settings like in Sri Lanka. Further, biosafety measures pertaining to SARS-CoV-2 need to be addressed in diagnosis (8. Infection control and prevention measures in both clinical and laboratory setting is another important aspect to prevent the spread of infection in Sri Lanka (9. Here, we describe how we promptly established a novel in-house RT-PCR method successfully to face the challenge in Sri Lanka.

\section{Public health response and its impact}

Methodology on verification of a molecular assay to detect SARS-CoV-2

This assay consists of two monoplex real-time RTPCR targeting highly conserved regions, $\mathrm{N}$ gene and
ORF1b gene of SARS-CoV-2 virus. The $\mathrm{N}$ gene was recommended as a screening assay and ORF1b gene as the confirmatory assay. The primer sequences were designed based on first published sequence data available at GenBank. Primer and probe sequence for ORF1b-nsp14 were: 5'-TGGGGYTTTACR GGTAACCT-3' forward primer, 5'-AACR 
CGCTTAACAAAGCACTC-3' reverse primer and 5'-FAM- TAGTTGTGA TGCWATCA TGACTAG-

TAMRA-3' probe. Primer and probe sequence for $\mathrm{N}$ gene were, 5'-TAATCAGACAAGGAACTGA TTA-3' forward primer, 5'-CGAAGG TGTGAC TTCCATG-3' reverse primer and 5'-FAM-GCAAA TTGTG CAATTTGCGG-TAMRA-3' probe. The expected amplicon sizes of ORF1b and $\mathrm{N}$ gene were $132 \mathrm{bp}$ and $110 \mathrm{bp}$, respectively. Assay was a previously validated and published, in-house RTPCR, which was developed by University of Hong Kong (10).

Primers and probes were delivered as a primer-probe premix, for ORF1b and $\mathrm{N}$ gene, separately and in the dried form. Reconstitution instructions were included in the protocol. Working primer-probe mix was aliquoted and stored at $-20^{\circ} \mathrm{C}$.

Positive controls which were plasmids of SARS virus for each target were delivered in the form of dried on filter paper. According to the protocol instructions, the positive control dilutions $10^{6}$ copies $/ \mu$ and $10^{4}$ copies $/ \mu \mathrm{l}$ were prepared, aliquoted and stored at $-20^{\circ} \mathrm{C}$ (10).

A verification run was performed to assess the assay performance. Three high positive controls $\left(10^{6}\right.$ copies $/ \mu \mathrm{l})$, three low positive controls $\left(10^{4}\right.$ copies $\left./ \mu \mathrm{l}\right)$ and three extracted negative controls along with a master mix control (no template control) were used for the verification run. Nucleic acid extraction was performed manually using a commercial kit (QIAamp RNA Mini kit, Qiagen). Recommended enzyme was TaqMan Fast Virus master mix, instead we used Invitrogen SuperScript III platinum one-step qRT-PCR kit. Assay was performed in ABI 7500 RTPCR platform while adhering to assay set up parameters and temperature profile (reverse transcription at $50^{\circ} \mathrm{C}$ for 5 minutes, inactivation of reverse transcriptase at $95^{\circ} \mathrm{C}$ for 20 seconds, 40 cycles of PCR amplification-Denaturing at $95^{\circ} \mathrm{C}$ for 5 seconds; annealing/extending at $58^{\circ} \mathrm{C}$ for 30 seconds) provided in the protocol (10).

Cycle threshold $(\mathrm{Ct})$ values of positive control dilutions of both genes were sent to the reference laboratory and were compatible with our values. Run validation criteria, master mix control, extracted negative controls without amplification curves and positive control with an exponential amplification curve need to be fulfilled to interpret results. Both $\mathrm{N}$ and ORF1b genes have exponential amplification curves taken as positive and both targets with no amplification curves as negative. If only one gene was positive, it was considered as indeterminate results and was referred to the WHO reference laboratory for further testing.

Fifteen samples (two positives -same patient and 13 negatives) tested were sent to WHO coronavirus reference laboratory, University of Hong Kong as per WHO recommendations for quality assurance. Results of NIC gave $100 \%$ concordance with reference laboratory results. According to the national/WHO case definition, respiratory samples from suspected patients with SARS-CoV-2 were investigated (11).

\section{Case definition -}

Patients with severe acute respiratory infection (fever, cough, and requiring admission to hospital) AND with no other etiology that fully explains the clinical presentation AND a history of travel to or residence in China during the 14 days prior to symptom onset,

\section{OR}

Patients with any acute respiratory illness AND at least one of the following during the 14 days prior to symptom onset:

close contact with a confirmed or probable case of 2019-nCoV infection, or

worked in or attend a health care facility where patients with confirmed or probable 2019-nCoV acute respiratory disease patients were being treated 
Lower respiratory samples were highly recommended and if not available. sputum samples were tested. Nasopharyngeal and oropharyngeal swabs were discouraged (11). Samples were transported in triple packing to NIC7 (9). They were unpacked in a BSL-2 level laboratory with BSL-2 level practices. WHO Biosafety Recommendations were adhered to during the processing of samples. A biosafety checklist was prepared, and the procedure was checked by the consultant. Local Biosafety Guidelines were also prepared based on WHO recommendations and taking consensus of the local authorities. Waste was disposed of after autoclaving. All staff was trained in handling hazardous material. Refresher course was given to reinforce biosafety practices (12).

Both genes were simultaneously employed to reduce the turnaround time. Further both cycle threshold values of both genes were serially assessed of the positive patient as the viral kinetics were ill defined in literature and as this is the first case. All samples that were not fitting to case definition for testing were subjected to influenza A and B assay (Altona RealStar commercial assay $\mathrm{GmBH}$ ). Negative samples were re-tested if clinical and travel/close contact history were highly suggestive of COVID-19. If swabs were negative for SARS-CoV-2 virus, lower respiratory samples were requested on the same day.

\section{Discussion}

Novel coronavirus laboratory diagnosis poses several challenges (10). At the time of the spread of COVID19 , there were no commercial assays available for detection of novel SARS-Cov-2. Only a few in-house methods were available. We were able to collaborate with the regional reference laboratory at University of Hong Kong (UHK) and procure the reagents and positive controls. Establishing a previously validated and published in-house assay was a challenge in a resource limited setting. One of the main challenges was the recommended enzyme for master mix that was not available in our laboratory. Another obstacle was preparing the positive controls which were dried on a filter paper. We did not have any experience and it was on the assumption that high positive and low positive viral concentration calculations were performed. However, we had the experience of detecting MERS coronavirus an in-house assay with collaboration of UHK in 2012, which was useful in setting up this task. We have performed CDC, USA in-house assays for influenza H1, H3, H5 and H7 and were familiar with biosafety practices pertaining to respiratory sample processing. Those practices and experience were beneficial in establishing a novel inhouse PCR assay to detect SARS CoV-2 within a very short period of time.

The prudence of introducing a detection method for SARS-Cov-2 was demonstrated the very next day (test established on 26 January and first laboratory confirmed case reported on 27 January) by identifying a positive patient from a Chinese traveller. Also, according to the case definition of COVID-19, several patient samples were tested in travellers from China and significant exposures to the positive patient including her husband and tourists in the same tourist group. None of the other suspected cases were positive.

Managing the limited resources in the laboratory while performing many other routine diagnostic assays was another major obstacle. Nevertheless, we were able to perform testing and reporting results within 36 hours of sample receiving in most of the instances. Starting from a limited capacity of testing 20 samples per day, we were able to increase it to 200 per day within 4 weeks; and when the real need arose, to 400 per day by dividing the staff into 3 teams, employing commercial assays and performing as many as 8 PCR runs per day till around 1.00 am due to the commitment and passion of the staff of Department of Virology, Medical Research Institute (MRI).

\section{Conclusion}

Laboratory confirmation of suspected COVID-19 cases are important in public health measures and in case management. However, there were some challenges to establish testing facility due to the novel nature of the infection and the non-availability of commercial assay as well as to obtain positive controls. Existing facilities and experience on MERS coronavirus and influenza PCR diagnostic capability in a WHO designated national influenza laboratory 
were useful in setting up an in-house assay, within a very short time. Existing biosafety measures that were placed for respiratory samples were very useful to handle for a novel respiratory viral infection.

\section{Author Declaration}

Acknowledgments: Dr Janaki Abeynayake (Head, Consultant Virologist, Department of Virology, $\mathrm{MRI)}$ for assistance and guidance and all staff members at Department of Virology, MRI.

Author contributions: VW wrote manuscript and did the local validation; DC designed the original work of the PCR assay; MP did the supervision of the original PCR assay and proofread the manuscript; JJ wrote manuscript, did the local validation and supervision of local tests.

\section{References}

1. WHO. Novel Coronavirus (2019-nCoV). Situation Report- 18, 7 February 2020. Available from: https://www.who.int/ docs/default-source/corona viruse/situation-reports/20200207-sitrep-18ncov.pdf? sfvrsn=fa644293_2.

2. Holshue ML, DeBolt C, Lindquist S, Lofy KH, Wiesman J, Bruce H, et al. First case of 2019 novel coronavirus in the United States. The New England Journal of Medicine 2020; 382: 929-936.

3. European Centre for Disease Prevention and Control. Risk assessment: outbreak of acute respiratory syndrome associated with a novel coronavirus, China; first cases imported in the EU/EEA. Second Update January 2020. Available from: https://www.ecdc.europa.eu/ en/publicationsdata/risk-assessment-outbreak-acute-respiratorysyndrome-associated-novel-0.

4. Jagodaararchchi R. Chinese workers settling down with ease. Sunday observer E paper, 15 July 2018. Available from: http://www. sundayobserver.lk.

5. Sri Lanka Tourism Development Authority. Monthly Tourist Arrivals Report - January 2020. Available from: https://www. sltda.gov.lk/monthly-touristarrivals-reports-2020.
6. Lai S, Bogoch I, Ruktanonchai N, Watts A, Li Y, Yu $\mathrm{J}$, Khan K. Assessing spread risk of Wuhan novel coronavirus within and beyond China, JanuaryApril 2020: a travel network-based modelling study. MedRxiv 9 March 2020. DOI: 10.1101/2020.02. 04.20020479.

7. WHO. Infection prevention and control during health care when novel coronavirus (nCoV) infection is suspected. Interim guidance. Available from: https://www.who.int/ publications/i/item/infectionprevention-and-control-during-health-care-whennovel-corona virus-(ncov)-infection-is-suspected20200125. Accessed 19 March 2020.

8. Reusken CBEM, Broberg EK, Haagmans B, Meijer A, Corman VM, Papa A, et al. Laboratory readiness and response for novel coronavirus (2019-nCoV) in expert laboratories in 30 EU/EEA countries. Euro Surveillance January 2020; 25(6): pii=2000082. Available from: https://doi.org/10.2807/15607917.ES. 2020.25.6.2000082

9. Centers for Disease Control and Prevention (CDC). Comprehensive Hospital Preparedness Checklist for Coronavirus Disease 2019 (COVID-19). Available from: https://www.cdc. gov/coronavirus/2019-ncov/ downloads/ HCW_Checklist_508.pdf. Accessed 25 March 2020.

10.Chu DKW, Pan Y, Cheng SMS, Hui KPY, Krishnan P, Liu Y, et al. Molecular diagnosis of a novel coronavirus (2019-nCoV) causing an outbreak of pneumonia. Clinical Chemistry 2020; 7: 1-7. DOI: 10.1093/clinchem/hvaa 02911.

11. Dawei Wang, Bo $\mathrm{Hu}$, Chang Hu, Fangfang Zhu, Xing Liu, Jing Zhang, BinbinWang, Hui Xiang, Zhenshun Cheng, Yong Xiong, Yan Zhao, Yirong Li, XinghuanWang, Zhiyong Peng. Clinical Characteristics of 138 hospitalized patients with 2019 novel coronavirus-infected pneumonia in Wuhan, China. JAMA 2020; 1-9. DOI: 10.1001/ jama.2020.1585.

12. Centers for Disease Control and Prevention (CDC). Interim Laboratory Biosafety Guidelines for Handling and Processing Specimens Associated with Coronavirus Disease 2019 (COVID-19), 2020. Available from: https://www.cdc.gov/corona virus/2019-nCoV/lab/lab-biosafety-guidelines.html. Accessed 17 February 2020. 